\title{
A Review of Recent Texture Classification: Methods.
}

\author{
M. Venkataramana, Asst.prof ,Prof .E.Sreenivasa Reddy, \\ Prof CH.Satyanarayana, S.Anuradha \\ 1 Asst.Prof, Dept Of CSE, Gitam University, Visakapatnama, India. ${ }^{2}$ Prof, Dept Of CSE Nagarjunauniversity, \\ ${ }^{3}$ Prof.Dept Of CSE Jntu Kakinada, India.4.Asst.Prof CSE Dept Gitam University.
}

\begin{abstract}
Texture classification is used in various pattern recognition applications that possess feature-liked Appearance. This paper aims to compile the recent trends on the usage of feature extraction and classification methods used in the research of texture classification as well as the texture datasets used for the experiments. The study shows that the signal processing methods, such as Gabor filters and wavelets are gaining popularity but old methods such as GLCM are still used but are improved with new calculations or combined with other methods. For the classifiers, nearest neighbor algorithms are still fairly popular despite being simple and SVM has become a major classifier used in texture classification. For the datasets, DynTex, Brodatz texture dataset is the most popularly used dataset despite it being old and with limited samples, other datasets are less used.

Index Terms: Texture Classification, wavelet-Based Dynamic, Computer Vision, Pattern Recognition, Machine Learning.
\end{abstract}

\section{Introduction}

Texture classification is the process to classify different textures from the given images. Although the classification of textures itself often seems to be meaningless in its own sense, texture classification can however be implemented a large variety of real world problems involving specific textures of different objects [1]. Some of the real world applications that involve textured objects of surfaces include rock classification [2], wood species recognition [3], face Detection [4], fabric classification [5], geographical landscape segmentation [6] and etc. All these applications allowed the target subjects to be viewed as a specific type of texture and hence they can be solved using texture classification techniques. Texture classification techniques are grouped up in five main groups in general, namely 1) structural; 2) statistical; 3) signal processing; 4) model-based stochastic [1], and; 5) morphology-based methods [7]. Out of the five groups, statistical and signal processing methods are the most widely used because they can be directly applied onto any type of texture. The rest are not as widely used because the structural methods need to implemented on structured textures which are naturally rare, the model based stochastic methods are not easily implemented due to the complexity to estimate the parameters and morphology-based methods are relatively new and the process are very simple, they may not promise very good textural features.

The main objective of this paper is to compile the recent trends in texture classification in terms of feature extraction and classification methods used as well as the texture datasets used in the training and testing process within the last five years. Section 2 shows the feature extraction methods used in the recent years. Section 3 shows the classification methods used in the recent years. Section 4 shows the popularly

\section{Feature Extraction Methods}

There are many different feature extraction methods that were introduced and used for texture classification problems. Most of these methods that were popularly used in recent years were statistical and signal processing methods.

\subsection{GLCM}

Grey Level Co-occurrence Matrices (GLCM) is an old feature extraction for texture classification that was proposed by Haralick et al. back in 1973 [8]. It has been widely used on many texture classification applications and remained to be an important feature extraction method in the domain of texture classification. It is a statistical method that computes the relationship between pixel pairs in the image. In the conventional method, textural features will be calculated from the generated GLCMs, e.g. contrast, correlation, energy, entropy and homogeneity [9]. However in recent years, the GLCM is often combined with other methods and is rarely used individually $[6,10$, and 12]. Other than the conventional implementation, there are a few other implementations of the GLCM, e.g. by introducing a second-order statistical method on top of the textural features in the original implementation [12], one-dimensional GLCM [13] and using the raw GLCM itself instead of the first-order statistics [14]. The GLCM can also be applied on different color space for color co occurrence matrix [15]. 


\subsection{Wavelets Methods}

Wavelet transforms is another signal processing method that have been implemented in image processing and pattern recognition for the last two decades [26]. It is currently an important feature to be used in texture classification and has been very popularly used [12, 27-42]. The Discrete Wavelet Transforms (DWT) is among the most popularly used wavelet transforms, some basic discrete wavelets include the Haar wavelet and Daubechies wavelets. Like the Gabor filters, the wavelet transform are preformed on the frequency domain of the images rather than the spatial domain. The information on the frequency domain is usually more stable than the spatial domain. Therefore, they often produces better features that leads to a higher accuracy despite being more complex and slower. Some other transforms that were used includes the curvelet transform [10, 18, 47-49] and the Wavelets based Dynamic Texture classification using Gumble Distribution [11] as well as a few other transforms that were less popularly used. E.g. ridge let transform [50, 51, 52], $\log$ polar transform [52], Radon transform [53] and etc.

\subsection{Independent Component Analysis (ICA)}

PCA had been used for feature extraction, but it has the limitation of only obtaining up to second-order statistics but ICA can overcome this problem and is capable of obtaining higher order statistics. [54]. It is used to separate a multivariate signal which is also implemented in texture classification but is not very popular [5457].

\subsection{Other Feature Extractions}

There are many other feature extractions that are not popularly used in recent years which some are recently proposed, including model-based stochastic methods, e.g. fractals [59, 60] and Markov random field [61, 62]. Also includes some other methods, e.g. Sequential Approximation Error Curves (SAEC) [63], Basic Image Features [64], Spectral Correlation Function (SCF) [65], Legendre Spectrum [66] and Multiscale Blob Features (MBF) [67].

\subsection{Summary and Findings on Feature Extractions}

It is easily noticeable that signal processing methods are very popularly used in the recent years, especially for Gabor filters and wavelets. Although these methods require more computation as they are examining the frequency domain, the accuracy obtained is good and usually outperform older and simpler techniques. The old technique like GLCM is however yet to be forgotten in the field of texture classification because it is one of the simplest textural feature which is old but is computationally inexpensive. It remains to be mainly used as a baseline algorithm for comparative studies especially when a new application of texture classification is experimented [5]. The GLCM is however more commonly used in some improved or combined ways recently but none of these variants have grown into a major trend. The major trend of the research today in terms of feature extraction for texture classification is accuracy oriented, however usually the newer algorithms that promises better accuracy is much more complicated in its calculations and often sacrifices the speed of the algorithm. The signal processing methods for example is a relatively slow algorithm with a higher accuracy [14]. Dynamic texture classification has attracted growing attention. Characterization of dynamic texture is vital to address the classification problem. The region covariance matrix is new in the area of texture classification. It has the potential to become the next trend due to its fast computations using integral images

\section{Classification Methods}

There are three major groups of classifiers are popularly used, including nearest neighbors, Artificial Neural Networks (ANN) and Support Vector Machines (SVM). Besides them, there are also other less popularly used classifiers or classification algorithms

\subsection{Nearest Neighbors}

The nearest neighbor algorithms are simple classifiers that select the training samples with the closest distance to the query sample. These classifiers will compute the distance from the query sample to every training sample and select the best neighbor or neighbors with the shortest distance. The k-Nearest Neighbor (k$\mathrm{NN}$ ) is a popular implementation where k number of best neighbors is selected and the winning class will be decided based on the best number of votes among the $\mathrm{k}$ neighbors [68]. The nearest neighbor is simple to be implemented as it does not require a training process. It is useful especially when there is a small dataset available which is not effectively trained using other machine learning methods that goes to the training process. However, the major drawback of the nearest neighbor algorithms is that the speed of computing distance will increase according to the number of training samples available[77]. 


\subsection{ANN}

ANNs are popular machine learning algorithms that were popular for the last decade and remains to be widely used until recent years. The basic form of ANN is the Multilayer Perception (MLP) which is a neural network that updates the weights through back-propagation during the training. It has proven to be useful in the past but is slowly losing popularity and is showing a trend of being taken over by the SVM that will be discussed in Section 3.3. Other variants of neural networks were also implemented in texture classification recently such as the Probabilistic Neural Network (PNN) [22, 69]. The Convolution Neural Network (CoNN) is a neural network that has convolution input layers that acts as a self learning feature extractor directly from the raw pixels of the input images. Therefore, it can perform both feature extraction and classification under the same architecture [70].

\subsection{SVM}

SVM are the newer trends in machine learning algorithm which is popular in many pattern recognition problems in recent years, including texture classification. SVM is designed to maximize the marginal distance between classes with decision boundaries drawn using different kernels [41]. SVM is designed to work with only two classes by determining the hyper plane to ivied two classes. This is done by maximizing the margin from the hyper plane to the two classes. The samples closest to the margin that were selected to determine the hyper plane is known as support vectors. Multiclass classification is also applicable, the multiclass SVM is basically built up by various two class SVMs to solve the problem, either by using one-versus-all or one-versusone. The winning class is then determined by the highest output function or the maximum votes respectively. This may cause the multiclass SVM to perform slower than the MLPs. Despite that, SVM is still considered to be powerful classifier which was replacing the ANN and has slowly evolved into one of the most important main stream classifier. They are now widely used in the research of texture classification.

\subsection{Other Classifiers}

Other classifiers are also used for texture classification but has yet to be popular in the recent years, e, g. the Bayes classifier [59, 71], Learning Vector Quantization (LVQ)[47] and Hidden Markov Model (HMM) [72].

\subsection{Summary and Findings on Classification Methods}

SVM is today not only the major trend in texture classification, it is also generally a very popular classifier in various pattern recognition problems, including recognition and detection problems. However it was not initially designed for multiclass problems, therefore it is adapted to implement of multiclass problem which is more complicated and will be slightly slower. As a comparison, the SVM has the best accuracy performance compared to ANN and nearest neighbors. But SVM and nearest neighbors are both required to store the sample points that helps to classify the problem space. In the nearest neighbors, all sample points have to be stored but in SVM, only the chosen samples which are of good representation to classify the problem space will be stored and known as support vectors. The ANN model however only requires the weights in the ANN model to be tune to represent the problem space, hence its needs in storing information is usually less than SVM and nearest neighbors. ANN and Bayes classifiers are often a classifier to be chosen due to their fast speed performance because their classification stages involved simpler calculations which helps to produce fast output results. The trend of the classifiers did not evolve specially for texture classification as it is generally following the trend of general machine learning. This showed that the texture classification does not require specific classifiers as compared to feature extractors. It is likely that the trend of usage for classifiers will continue to follow the major trends of machine learning.

\section{Texture Datasets}

There are a number of texture datasets that were used in experiments on texture classification, e.g. the Brodatz texture album and CUReT texture dataset which were more widely used.

\subsection{Brodatz Texture Album}

The Brodatz textures are popular and widely used as benchmark datasets in texture classification. It is consists of 112 textures that were abstracted from the Brodatz texture album [73]. Each of these textures is produced from a single image scanned from the texture album.

Although the Brodatz textures are widely used, there are many different subsets of the dataset which often involves only part of the album and some with rotated and scaled samples added [74]. The entire dataset are sometimes also used [75]. Sample images of the Brodatz textures are Shown in Figure 4.1. 


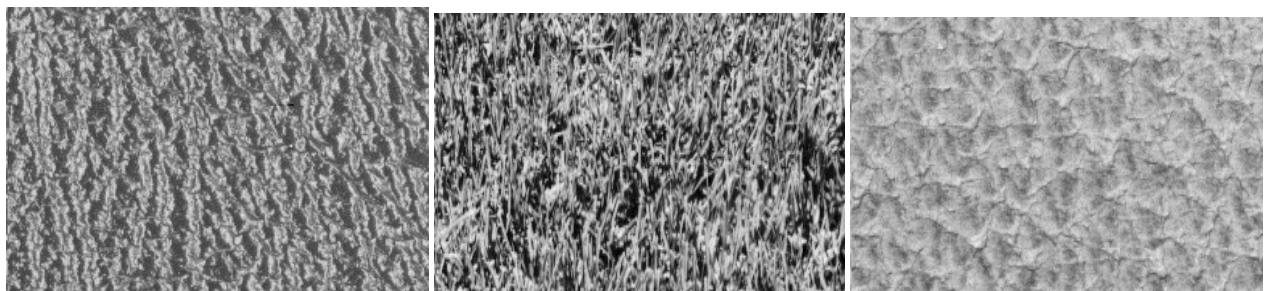

Fig 4.1. Samples of four images from the Brodatz texture album.

The main limitation of the Brodatz texture dataset is that all the textures are represented by a single image only. Therefore, the users need to segment to dataset into smaller segments, and often scale and rotate them. Since there is only a single image for each texture, the segments of the same texture will be rather homogeneous for both training and testing

\subsection{CUReT Dataset}

The Columbia-Utrecht Reflectance and Texture (CUReT) dataset is produced in a collaborated research between Columbia University and Utrecht University [76]. The dataset includes 61 different textures with 92 images for each class. These images are acquired under different illuminations and viewing directions [77]. With the differences in illuminations and viewing directions, this dataset creates greater challenge to the algorithm which should tackle the problem on illumination and direction since both of these factors could cause the samples to be showing different appearance, unlike the Brodatz dataset that only provide one single illumination and viewing direction for each texture. Sample images of the CUReT textures are shown in Figure 4.2 .
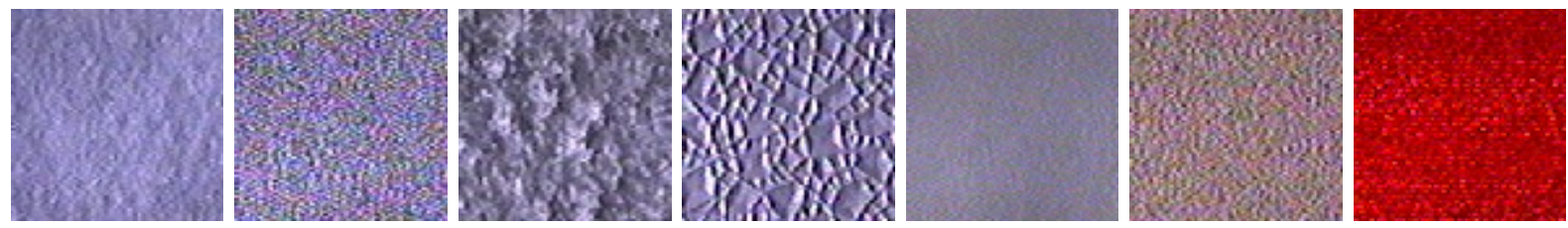

Fig 4.2. Samples of images from the CUReT Texture album.

\subsection{VisTex Dataset}

The Vision Texture (VisTex) dataset is prepared by the Massachusetts Institute of Technology (MIT) [78]. The dataset is not only consisting of homogeneous frontal acquisition of textures, it also comes with realworld scenes with multiple textures and video textures. This dataset is not very popularly used but more frequent than those in Section 4.4. Sample images of the dataset are shown in Figure 4.3.
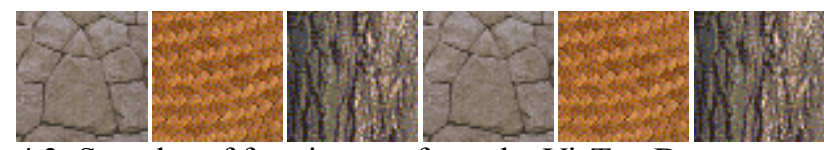

Fig 4.3. Samples of four images from the VisTex Dataset textures

\subsection{DynTex Dataset}

The DynTex database is a diverse collection of high-quality dynamic texture videos. Currently we are finalizing the structure of the database.

Dynamic textures are typically result from processes such as of waves, smoke, fire, a flag blowing in the wind, a moving escalator, or a walking crowd. Many real-world textures occurring in video databases are dynamic and retrieval should be based on both their dynamic and static features. Important tasks are thus the detection, segmentation and perceptual characterization of dynamic textures. Sample images of the dataset are shown in Figure 4.4.
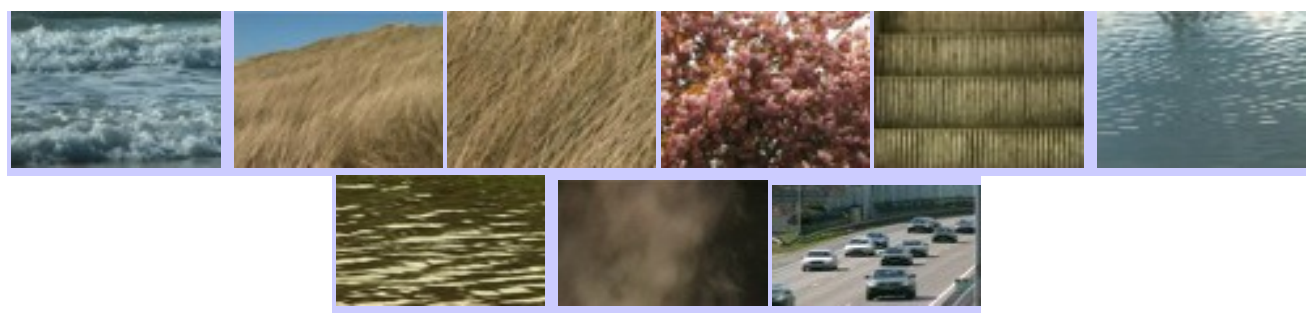

Fig 4.4. Samples of four images from the DynTex textures 


\subsection{Other Datasets}

There are a few other datasets that were less popularly used, e.g. the MeasTex [19], PhoTex [32], OuTex [19, 47], KTH-TIPS [64, 77] and UIUCTex [60, 64, and 77].

\subsection{Summary and Findings on Texture Datasets}

The common problem of texture classification today is that texture datasets that are available in the field are usually having each textures acquired only once, therefore each class often have very homogeneous training and testing samples. This problem has not been solved due to the difficulty in preparing datasets with different acquisition of a same type of texture for all the textures. The Brodatz texture album is a printed album that is available backed in 1996. The scanned textures are then popularly used in the research of texture classification as a popular benchmark dataset. Other newer datasets have yet to be very popularly used but has often included more aspect, such as variations in viewing angle and illumination. Some of this dataset, e.g. the VisTex, DynTexis still being improved as video textures are planned to be included in the future [78]. As the Brodatz dataset has currently been used as a dominant benchmark dataset, in the future, the other datasets are likely to gain popularity as the trend of research will likely to move from accuracy-oriented towards tackling the issue on viewing angles and illuminations. Datasets with multiple instants of the same texture acquired from different materials will also be useful for future research in handling the variances within the textures itself.

\section{Conclusion}

In this paper, the trend of usage in the feature extraction methods, classification methods and texture datasets in the last five years is discovered. For the feature extraction methods, wavelet transforms and other signal processing methods are among the most popularly used feature extraction due to their promising accuracy. Surprisingly, old methods such as GLCM are still used but their implementations are improved or combined with other methods. In terms of the classification method, SVM has took over ANN as the most commonly used classifier which has also proven to be able to outperform the ANN in terms of accuracy. For the experimental datasets, variants of the Brodatz texture datasets remained to be the most popular benchmark dataset in the research of texture classification. The trend of the usage for the feature extraction and classification method showed that the researches are mainly accuracy-oriented, where the signal processing methods and SVM could produce a better accuracy but these methods are often more complex than older methods and could not guarantee a better speed performance. The increase in computational capabilities of the computers today has assisted in the growth of the research by allowing more complex algorithms to work within a reasonable time. However, when a computer with lower computational power is being concerned, e.g. an embedded platform, an older yet simpler method is often more useful [14]. As smart phones and other compact devices are gaining popularity, texture classification-based applications that can be run on these platforms will require higher efficiency in terms of speed while at the same time offering satisfying accuracy. Although texture classification has been studied for decades, it has yet to come to an end as newer studies has revealed that there are simply much more space for the research to carry on especially since it is generally useful to solve many different real world problems. These aspects that could be focused on in future research include the speed and required storage as well as the varying acquisition conditions. With the research on improving the accuracy being carried out for years and is slowly moving against its bottleneck, the perspective on solving illumination and viewing angle problem, searching for a balance between speed and accuracy are likely to become more important to be studied in the years to come.

\section{References}

[1] M. Tuceryan, and A.K. Jain, "Texture Analysis," The Handbook of Pattern Recognition and Computer Vision (2 ${ }^{\text {nd }}$ Ed), World Scientific Publishing, Singapore, pp. 207-248, 1998.

[2] L. Lepisto, L. Kunttu, J. Autio, and A. Visa, "Rock Image Classification Using Non-Homogenous Textures and Spectral Imaging," WSCG, 2003.

[3] J.Y. Tou, Y.H. Tay, and P.Y. Lau, "A Comparative Study for Texture Classification Techniques on Wood Species Recognition Problem," ICNC, vol. 5, pp. 8-12, 2009.

[4] W.H. Yap, M. Khalid, and R. Yusof, "Face Verification with Gabor Representation and Support Vector Machines," AMS, pp. 451459,2007

[5] Y.B. Salem, and S. Nasri, "Texture Classification of Woven Fabric Based on a GLCM Method and using Multiclass Support Vector Machine," SSD, 2009.

[6] J.A.R. Recio, L.A.R. Fernandez, and A. Fernandez-Sarria ,"Use of Gabor Filters for Texture Classification of Digital Images," Física de la Tierra, no. 17, pp. 47-56, 2005.

[7] Y.Q. Chen, "Novel Techniques for Image Texture Classification,” PhD Thesis, University of Southampton, United Kingdom, 1995.

[8] R.M. Haralick, K. Shanmugam, and L. Dinstein, "Textural Features for Image Classification,” IEEE TSMC, vol. 3, pp. 610-621, 1973.

[9] M. Petrou, and P.G. Sevilla, Image Processing Dealing with Texture, John Wiley \& Sons, West Sussex, England, 2006.

[10] S. Arivazhagan, L. Ganesan, and T.G.S. Kumar, "Texture Classification using Curvelet Statistical and Co-occurrence Features," ICPR, 2006. 
[11] Yu-Long Qiao, Chun-Yan Song, and Fu-Shan Wang "Wavelet-Based Dynamic Texture Classification Using Gumbel Distribution" Mathematical Problems in Engineering Volume 2013 (2013).

[12] M.B. Othmen, M. Sayadi, and F. Fnaiech, "Interest of the Multi-Resolution Analysis Based on the Co-occurrence Matrix for Texture Classification," IEEE MELECON, pp. 852-856, 2008.

[13] J.Y. Tou, Y.H. Tay, and P.Y. Lau, “One-dimensional Greylevel Co-occurrence Matrices for Texture Classification,” ITSIM, vol. 3, pp. 1592-1597, 2008.

[14] J.Y. Tou, K.K.Y. Khoo, Y.H. Tay, and P.Y. Lau, "Evaluation of Speed and Accuracy for Comparison of Texture Classification Implementation on Embedded Platform," IWAIT, 2009.

[15] A. Porebski, N. Vandenbroucke, and L. Macaire, "Iterative Feature Selection for Color Texture Classification," ICIP, no. 3, pp. 509-512, 2007.

[16] T. Ojala, and M. Pietikainen, “Unsupervised Texture Segmentation Using Feature Distributions,” Pattern Recognition, vol. 32, pp. 477-486, 1999.

[17] T. Maenpaa, M. Pietikainen, and T. Ojala, “Texture Classification by Multi-Predicate Local Binary Pattern Operators," ICPR, vol. 3, pp. 3951-3954, 2000.

[18] Y. Shang, W. Hou, R. Wu, and Z. Meng, “Antinoise ,Rotation Invariant Texture Classification Based on LBP Features of Dominant Curvelet Subbands," IIT A, pp. 365-369, 2008.

[19] S. Liao, M.W.K. Law, A.C.S. Chung, "Dominant Local Binary Patterns for Texture Classification," IEEE TIP, vol. 18, no. 5, pp. $1107-1118,2009$.

[20] J.Y. Tou, Y.H. Tay, and P.Y. Lau, "Gabor Filters as Feature Images for Covariance Marix on Texture Classification Problem," ICONIP 2008, vol. 5507, pp. 745-751, 2009.

[21] X. Xie, J. Gong, Q. Dai, and F. Xu, "Rotation and Scaling Invariant Texture Classification Based on Gabor Wavelets," VIE, pp. 393-396, 2008.

[22] N. Qaiser, M. Hussain, A. Hussain, N. Iqbal, and N. Qaiser, "Dissimilarity Analyst of Signal Processing Methods for Texture Classification," IEEE ICEIS, 2006.

[23] R. Muniz, J.A. Corrales, M. Rico-Secades, "Use of Band Ratioing for Building Illumination Independent Texture Classification Systems," ICPR, 2008.

[24] U.A. Ahmad, K. Kidiyo, and R. Joseph, "Texture Features Based on Fourier Transform and Gabor Filters: An Empirical Comparison," ICMV, pp. 67-72, 2007.

[25] M. Jian, S. Chen, and J. Dong, "Illumination-Invariant Texture Classification Based on Self-Similarity and Gabor Wavelet," IITA, pp. 352-355, 2008.

[26] I. Daubechies, "Orthonormal Bases of Compactly Supported Wavelets," Communications on Pure and Applied Mathematics, vol. 41, pp. 909-996, 1988.

[27] T. Su, F. Kung, and Y. Kuo, "Application of BackPropagation Neural Network Fuzzy Clustering in Textile Texture Automatic Recognition System,” ICWAPR, pp. 46-49, 2008.

[28] M. Liu, Y. Hou, X. Zhu, D. Yang, and X. Meng, "New Approach for Texture Classification Based on Concept,” CISP, pp. 160-164, 2008.

[29] Z. Wang, and J. Yong, "Texture Analysis and Classification with Linear Regression Model Based on Wavelet Transform," IEEE TIP, vol. 17, no. 8, pp. 1421-1430, 2008.

[30] A. Jalil, I.M. Qureshi, A. Manzar, and R.A. Zahoor, "Rotation-Invariant Features for Texture Image Classification,IEEE ICEIS, 2006.

[31] Y. Qiao, and S. Sun, “Texture Classification Using Wavelet Frame Representation Based Feature,” IEEE ICEIS, 2006.

[32] J. Dong, Y. Duan, and Z. Yang, "Three-dimensional Surface Texture Classification Based on Support Vector Machines and Wavelet Packets," IITA, pp. 124-127, 2008.

[33] Y. Huang, L. Wang, C. Li, "Texture Analysis of Ultrasonic Liver Image Based on Wavelet Trans form and Probabilistic Neural Network,” BMEI 2008, pp. 248-252, 2008.

[34] Y. Qiao, C. Song, C. Zhao, "Double-Density Discrete Wavelet Transform Based Texture Classification,” IIH-MSP, vol. 1, pp. 9194, 2007.

[35] N. Dasgupta, and L. Carin, “Texture Analysis with Variational Hidden Markov Trees,” IEEE TSP, vol. 54, no. 6, pp. 2352-2356, 2006.

[36] Q. Wang, H. Li, and J. Liu, "Subset Selection using Rough Set in Wavelet Packet Based Texture Classification," ICWAPR 2008, pp. 662-666, 2008.

[37] S. Liu, and P. Qu, "Fabric Texture Classification Based on Wavelet Packet," ICEMI, pp. 238-241, 2007.

[38] Y. Qia, and S. Sun, "Texture Classification Using Modulus Extreme of Wavelet Frame Representation," CIS, vol. 2, pp. 1775-1778, 2006.

[39] E.P. Lam, "Texture Classification Using Wavelet Decomposition,” IEEE SoSE, 2008.

[40] K. Huang, and S. Aviyente, "Wavelet Feature Selection for Image Classification,” IEEE TIP, vol. 17, no. 9, pp. 1709-1720, 2008.

[41] C. Chen, C. Chen and C. Chen, "A Comparison of Texture Features Based on SVM and SOM,” ICPR, vol. 2, pp. 630-633, 2006.

[42] S. Selvan, and S. Ramakrishnan, "SVD-Based Modeling for Image Texture Classification Using Wavelet Transformation," IEEE TIP, vol. 16, no. 11, pp. 2688-2696, 2007.

[43] R. Javidan, M.A. Masnadi-Shirazi, Z. Azimifar, and M.H. Sadreddini, "A Comparative Study between Wavelet and Contourlet Transform Features for Textural Image Classification,” ICTTA, 2008.

[44] Z. Long, and N.H. Younan, "Contourlet Spectral Histogram for Texture Classification,” IEEE SSIAI, pp. 31-35, 2006.

[45] Z. Liu, "Minimum Distance Texture Classification of SAR Images in Contourlet Domain," CSSE, pp. 834-837, 2008.

[46] S. Li, X. Fu, B. Yang, "Nonsubsampled Contourlet Transform for Texture Classifications using Support Vector Machines," IEEE ICNSC, pp. 1654-1657, 2008.

[47] A.D. Lillo, G. Motta, J.A. Storer, "Multiresolution Rotation-Invariant Texture Classification Using Feature Extraction in the Frequency Domain and Vector Quantization,” DCC, pp. 452-461, 2008.

[48] L. Semler, and L. Dettori, "Curvelet-based Texture Classification of Tissues in Computed Tomography," ICIP, pp. 2165-2168, 2006.

[49] Y. Shang, Y. Diao, and C. Li, "Rotation Invariant Texture Classification Algorithm Based on Curvelet Transform and SVM," ICMLC, pp. 3032-3036, 2008.

[50] G.Y. Chen, and P. Bhattacharya, "Invariant Texture Classification Using Ridgelet Packets,” ICPR, 2006.

[51] K. Huang, and S. Aviyente, "Rotation Invariant Texture Classification with Ridgelet Transform and Fourier Transform," ICIP, pp. 2141-2144, 2006. 
[52] H. Mahersia, and K. Hamrouni, “New Rotation Invariant Features for Texture Classification,” ICCCE, pp. 687-690, 2008.

[53] G. Liu, Z. Lin, and Y. Yu, "Radon Representation-Based Feature Descriptor for Texture Classification," IEEE TIP, vol.18, no. 5, pp. 921-928, 2009.

[54] D.A.A. Nadi, and A.M. Mansour, "Independent Component Analysis (ICA) for Texture Classification," SSD, 2008.

[55] S. Chindaro, K. Sirlantzis, and M.C. Fairhurst, "ICA-Based Multi-Colour Space Texture Classification System," Electronic Letters, vol. 42 , no. $21,2006$.

[56] H.L. Borgne, A. Guerin-Dugue, and N.E. O’Connor, "Learning Midlevel Image Features for Natural Scene and Texture Classification," IEEE TCSVT, vol. 17, no. 3, pp. 286-297, 2007.

[57] D. Coltuc, T. Fournel, and J. Becker, "Texture Classification by ICA," ISSCS, 2007.

[58] O. Tuzel, F. Porikli, and P. Meer, "Region Covariance: A Fast Descriptor for Detection and Classification," ECCV, vol. 1, pp. 697$704,2006$.

[59] J.H. Kim, S.C. Kim, and T.J. Kang, "Fractal Dimension Cooccurrence Matrix Method for Texture Classification,” IEEE TENCON, 2006.

[60] M. Varma, and R. Garg, "Locally Invariant Fractal Features for Statistical Texture Classification,” IEEE ICCV, pp. 1-8, 2007.

[61] X. Bai, and K. Wang, "Research on Classification of Wood Surface Texture Based on Markov Random Field," ICIEA, pp. 664-668, 2007.

[62] Y. Zhao, L. Zhang, P. Li, and B. Huang, "Classification of High Spatial Resolution Imagery Using Improved Gaussian Markov Random-Field-Based Texture Features,” IEEE TGRS, vol. 45, no. 5, pp. 1458-1468, 2007.

[63] J.G. Rosiles, S. Upadhyayula, and S.D. Cabrera, "Rotationally-Blind Texture Classification using Frame Sequential Approximation Error Curves," IEEE ICASSP, pp. 1325-1328, 2008.

[64] M. Crosier, and L.D. Griffin, "Texture Classification with a Dictionary of Basic Image Features," 2008.

[65] M.C. Amirani, and A.A.B. Shirazi, “Texture Classification Using Cyclic Spectral Function,” CISP 2008, pp. 834-838, 2008.

[66] M. Abadi, and E. Grandchamp, "Legendre Spectrum for Texture Classification," ICSP, vol. 2, 2006.

[67] Q. Xu, and Y.Q. Chen, "Multiscale Blob Features for Gray Scale, Rotation and Spatial Scale Invariant Texture Classification," ICPR, 2006.

[68] B.D. Ripley, Pattern Recognition and Neural Networks, Cambridge University Press, United Kingdom, 1996.

[69] G. Yu, S.V. Kamarthi, "Texture Classification Using Wavelets with a Cluster-Based Feature Extraction,” ISSCAA, 2008.

[70] F.H.C. Tivive, and A. Bouzerdoum, "Texture Classification using Convolutional Neural Networks," IEEE TENCON, 2006.

[71] Y. Xu, M. Sonka, G. McLennan, J. Guo, and E.A. Hoffman, "MDCT-Based 3-D Texture Classification of Emphysema and Early Smoking Related Lung Pathologies,” IEEE TMI, vol. 25, no. 4, pp. 464-475, 2006.

[72] V.N. Vasyukov, and N.V. Sysoev, "Texture Image Segmentation Using Multiscale Wavelet-Domain Hidden Markov Model," IFOST, pp. 151-153, 2007.

[73] P. Brodatz, Textures: A Photographic Album for Artists and Designers, Dover, New York, 1996.

[74] T. Ojala, M. Pietikainen, and J. Kyllonen, "Gray Level Coocuurence Histograms via Learning Vector Quantization," SCIA, pp. 103-108, 1999.

[75] R.W. Picard, T. Kabir, and F. Liu, "Real-time Recognition with the Entire Brodatz Texture Database,” IEEE CVPR, pp. 638-639, 1993.

[76] http://www1.cs.columbia.edu/CAVE/software/curet [77] S. Kondra, and V. Torre, "Texture Classification Using Three Circular Filters," ICVGIP, pp. 429-434, 2008.

[77] Jing Yi Toul, Yong Haur Tayl, Phooi Yee Lau "RECENT TRENDS IN TEXTURE CLASSIFICATION: A REVIEW", Symposiumon Progress in Information \& Communication Technology 2009 